Revista de Matemática: Teoría y Aplicaciones 2007 14(1) : 1-6

CIMPA - UCR - CCSS ISSN: 1409-2433

\title{
THE BLACK-SCHOLES TYPE FINANCIAL MODELS AND THE ARBITRAGE OPPORTUNITIES
}

\author{
NikOlay SukHOMLiN*
}

Recibido/Received: 15 Jul 2006 - Aceptado/Accepted: 31 Jan 2007

\begin{abstract}
By using the conservation laws concept, we study certain financial models similar to the Black-Scholes model. We show that without complement limitations such models can have two or more volatilities. This fact imposes several intrinsic limitations for the dynamical system parameters in order to guarantee the correct definition.
\end{abstract}

Keywords: Black-Scholes model, volatility, laws of conservation.

\section{Resumen}

Usando el concepto de leyes de conservación, estudiamos ciertos modelos financieros similares al modelo de Black-Scholes. Demostramos que sin limitaciones complementarias tales modelos pueden tener dos o más volatilidades. Este hecho impone varias limitaciones intrínsecas para los parámetros de sistemas dinámicos con fines de garantizar la definición correcta de dichos sistemas.

Palabras clave: Modelo de Black-Scholes, volatilidad, leyes de conservación.

Mathematics Subject Classification: C12, C65.

\section{Introduction}

The classical solution to the Black-Scholes Equation has the following structure:

$$
V(t, S)=S N\left(d_{1}\right)-F(t) N\left(d_{2}\right)
$$

\footnotetext{
*Department of Physics, the Autonomous University of Santo-Domingo (UASD), Ciudad Universitaria, Ap. 1000, Santo Domingo Dominican Republic, Phone: 80968631 19; and Department of Economics, the Pontifical Catholic University of Santo-Domingo (PUCMM), Ave. Abraham Lincoln, Ap. 2748, Santo Domingo, Dominican Republic; Phone 809535 0111, ext 2144, Fax: 809534 7060. E-Mail: w17971865@gmail.com, ww17971865@yahoo.fr
} 
with:

$$
\begin{gathered}
F(t)=K e^{-r(T-t)}, N(d)=\frac{1}{\sqrt{2 \pi}} \int_{-\infty}^{d} e^{-u^{2} / 2} d u \\
d_{2}=\frac{\ln (S / K)}{\tau}-\beta \tau \\
d_{1}=d_{2}+\tau \\
\beta=\frac{1}{2}-\frac{r}{\sigma^{2}}, \tau=\sigma \sqrt{T-t}
\end{gathered}
$$

then

$$
S \frac{d N\left(d_{1}\right)}{d\left(d_{1}\right)}=F(t) \frac{d N\left(d_{2}\right)}{d\left(d_{2}\right)}
$$

where $V$ is the value of the call option (theoretical call premium), $S$ the current stock price at the moment of time $t, r$ is the risk-free interest rate, and $\sigma$ is the volatility (the last two parameters being supposed constant). The condition at the boundaries is: $V=\max (S-K ; 0)$. The constants $K$ and $T$ respectively represent the strike price and the option expiration date.

This is a well known relation for the Black-Scholes classical model (see for example [11]).

Let be the function:

$$
\xi=V^{(2)}-V^{(1)} ; V^{(1)}=\partial V / \partial(\ln S), V^{(2)}=\partial^{2} V / \partial(\ln S)^{2} .
$$

Using the cumulative normal distribution function property $\frac{d^{2} N(x)}{d x^{2}}=-x \frac{d N(x)}{d x}$ we obtain: $\xi=\frac{1}{\tau} S \frac{d N\left(d_{1}\right)}{d\left(d_{1}\right)}$. Let the elasticity of the auxiliary function $\xi$ be:

$$
E_{\xi}=\frac{\partial(\ln \xi)}{\partial(\ln S)}=-\frac{1}{\tau^{2}} \ln (S / K)+\beta
$$

hence

$$
\tau^{2}\left(E_{\xi}-\beta\right)-\ln (S / K)=0 .
$$

After the substitution of $\beta$ and $\tau$ from expression (1) in expression (4), we express the volatility as a function of market variables:

$$
\sigma^{2}=\frac{\ln (K / S)-r(T-t)}{(T-t)\left(E_{\xi}-1 / 2\right)}
$$

The verification of this formula by simulation (see [8], [9] and [10]) shows the $10^{-9}$ order of precision. The expression 4 represents the conservation law correspondent to the classic Black - Scholes solution (see [7]):

$$
\tau^{2}\left(E_{\xi}-\beta\right)+\ln (S)=\ln (K)=\text { const. }
$$

Thus one expression that "conserves" its value during the dynamical system evolution is constructed. It is possible to proceed in the same way with different expressions similar to 
the Black - Scholes classic formula. For example, [1] deduce an expression for the Value of a European Constant Underlying Elasticity in Strikes (CUES) call Option that generalizes the Black-Scholes classic solution:

$$
C(t, S)=e^{-\varepsilon \tau^{2}} S N\left(d_{1}\right)-F(t, S) N\left(d_{2}\right)
$$

with:

$$
\begin{aligned}
K & =q^{1 /(1-\alpha)} \\
F(t, S) & =K(S / K)^{\alpha} \exp \left[\left(-\alpha \varepsilon-(\alpha-1) \beta+\left(\alpha^{2}-1\right) / 2\right) \tau^{2}\right] \\
\varepsilon & =\delta / \sigma^{2} \\
d_{1} & =\frac{\ln (S / K)}{\tau}+(1-\beta-\varepsilon) \tau \\
d_{2} & =d_{1}+(\alpha-1) \tau \\
\beta & =\frac{1}{2}-\frac{r}{\sigma^{2}} \\
\tau & =\sigma \sqrt{T-t}
\end{aligned}
$$

and $N($.$) is a cumulative normal distribution function. By simple calculus we obtain a$ formula equivalent to expression (2):

$$
e^{-\varepsilon \tau^{2}} S \frac{d N\left(d_{1}\right)}{d\left(d_{1}\right)}=F(t, S) \frac{d N\left(d_{2}\right)}{d\left(d_{2}\right)}
$$

Now the correspondent to characteristic $\xi$ for the CUES problem is:

$$
\xi=C^{(2)}-(1+\alpha) C^{(1)}+\alpha C=\frac{1-\alpha}{\tau} e^{-\varepsilon \tau^{2}} S \frac{d N\left(d_{1}\right)}{d\left(d_{1}\right)} .
$$

Its elasticity is a linear function of $\ln (S / K)$ :

$$
E_{\xi}=\frac{\partial(\ln \xi)}{\partial(\ln S)}=-\frac{1}{\tau^{2}} \ln (S / K)+\beta+\varepsilon .
$$

The conservation law for the value of an European CUES call Option appears in similar form:

$$
\tau^{2}\left(E_{\xi}-\beta-\varepsilon\right)+\ln (S)=\ln (K)=\text { const. }
$$

It is easy to verify that all formulas from expression (5) to expression (9), transform into correspondent formulas for the classic Black-Scholes solution under the conditions: $\alpha \rightarrow 0, \varepsilon \rightarrow 0$. So all results established for the Black - Scholes model can be automatically extended to the Blenman and Clark model (see [1]). Particularly the volatility for this model is:

$$
\sigma^{2}=\frac{\ln q /(1-\alpha)-\ln (S)-r(T-t)}{(T-t)\left(E_{\xi}-\varepsilon-1 / 2\right)} .
$$

Using this expression it is possible to verify the reliability of the the model at [1] by calculus with market data. 
The situation is more complicated in the case of the Valuation of Defaultable Bonds Using Signaling Process Model (see [2]) and its extension (see [5] and [6]). The solution of a Black-Scholes type equation is:

$$
P(t, S)=G(t) S N\left(d_{1}\right)-F(t, S) N\left(d_{2}\right)+\text { a function of time }
$$

with:

$$
\begin{aligned}
\alpha-\sigma^{2} / 2 & =\sigma^{2} \nu, \\
m & =-2(1-\beta) \nu, \\
\tau & =\sigma \sqrt{t}, \\
d_{1} & =\frac{\ln \left(S / S_{0}\right)}{\tau}+\nu \tau, \\
d_{2} & =-d_{1}-m \tau, \\
F(t, S) & =G\left(S / S_{0}\right)^{m} e^{\beta \nu m \tau^{2}},
\end{aligned}
$$

$\alpha, \beta, \sigma, S_{0}=$ const, $N($.$) is a cumulative normal distribution function, and G(t)$ is certain function on time. The parameter $\beta$ characterizes the distinction between the Lo and Hui model $(\beta \neq 0)$ and the Cathcart and El-Jahel model (see $[2])(\beta=0)$. By simple calculus we obtain a formula equivalent to expression 2 :

$$
G \frac{d N\left(d_{1}\right)}{d\left(d_{1}\right)}=F(t, S) \frac{d N\left(d_{2}\right)}{d\left(d_{2}\right)}
$$

The equivalent of the auxiliary characteristic in expression 3 is:

$$
\xi=P^{(2)}-m P^{(1)}=-\frac{2 d_{1}+m \tau}{\tau^{2}} G \frac{d N\left(d_{1}\right)}{d\left(d_{1}\right)} .
$$

In this case the elasticity $E_{\xi}$ is a rational function of $\ln \left(S / S_{0}\right)$ :

$$
E_{\xi}=\frac{\xi^{(1)}}{\xi}=-\frac{2 d_{1}^{2}+m d_{1}-2}{\tau\left(2 d_{1}+m \tau\right)} .
$$

Entonces:

$$
A B \tau^{2}+(A+B) \ln \left(S / S_{0}\right)-1+\tau^{-2} \ln ^{2}\left(S / S_{0}\right)=0
$$

with:

$$
A=E_{\xi}+\nu, B=\beta \nu
$$

We conclude two cases emerge:

1. If $\beta=0$ (the Cathcart and El-Jahel model, see [2]) one expression for the volatility exists:

$$
\sigma^{2}=t^{-1} \ln ^{2}\left(S / S_{0}\left[1-A \ln \left(S / S_{0}\right)\right]^{-1}\right.
$$

with evident restriction for the signaling variable: $A \ln \left(S / S_{0}\right)<1$. 
2. If $\beta \neq 0$ (Lo and Hui model, see [5] and [6]) there are two expressions for the volatility under the conditions $A B>0,1>(A+B) \ln \left(S / S_{0}\right)$ :

$$
\left(\sigma^{2}\right)_{1,2}=\frac{1}{A B t}\left[1-(A+B) \ln \left(S / S_{0}\right) \pm \sqrt{(A-B)^{2} \ln ^{2}\left(S / S_{0}\right)-2(A+B) \ln \left(S / S_{0}\right)+1}\right] .
$$

To obtain only one expression it is necessary to change the conditions, for example to impose $A B<0$.

We can conclude that without certain restrictions the existence of two volatilities is intrinsic for the Lo and Hui Valuation of Defaultable Bonds Using Signaling Process Model and it is not in the case of the Cathcart and El-Jahel model. Lo and Hui introduce the parameter $\beta$ with the goal to approach to real market data. With such introduction appears the possibility of model's indeterminism. Another example can be found in [3] and [4]. The authors use the Black and Scholes option pricing technique to develop an expression for the Pricing of Defaultable Bonds with Log-Normal Spread. By similar calculus we obtain a cubic equation with three solutions under certain conditions:

$$
\begin{gathered}
{[\lambda(T-t) \ln (h / H)] \sigma^{3}+\left[1-\left(E_{\xi}-\frac{1}{2}\right) \ln (h / H)\right](T-t) \sigma^{2}-\left[\ln ^{2}(h / H)-\mu \ln (h / H)\right]=0} \\
\lambda, T, H, \sigma, \mu=\text { const. }
\end{gathered}
$$

We can conclude that without specific restrictions the existence of two or tree volatilities is intrinsic for the Cané de Estrada and all model (see [3] and [4]).

\section{Conclusion}

We show that it is possible to express the volatility for the Black and Scholes classic model in function of market data. It is true in framework to this model. One expression for the volatility and one conservation law exist. We find the important role of the elasticity in this model too.

For other models the situation can be complicated: our procedure suggests the conclusion that several volatilities and conservation laws can exist.

We studied two concrete cases and found that under certain conditions two and three volatilities exist. Without specific restrictions the existence of several volatilities is intrinsic for the models mentioned.

It is possible to consider that the existence of two or more volatilities is related to the presence of arbitrage opportunities and that the restrictions to have only one volatility are related to the arbitrage absence.

\section{Acknowledgements}

Author is grateful to SEESCyT foundation, to Mr. Roberto Reyna and to the UASD Department of Physics for their cooperation. I am especially grateful to the Editor in chief Dr. Javier Trejos of the Revista de Matemática: Teoría y Aplicaciones for his useful observations. Also, the author expresses his gratitude to Dr. Alemán and to PUCMM Department of Economics. 
N. SUKHOMLIN Rev.Mate.Teor.Aplic. (2007) 14(1)

\section{References}

[1] Blenman, L.P.; Clark, S.P. (2005) "Options with constant underlying elasticity in strikes", Review of Derivatives Research 8: 67-83.

[2] Cathcart, L.; El-Jahel, L. (1998) "Valuation of defaultable bonds", Journal of Fixed Income 8: 65-78.

[3] Cané de Estrada, M.; Cortina, E.; Ferro Fontán, C.; Di Fiori, J. (2001) "Defaultable bonds with log-normal spread: an application of the model to Argentinean and Brazilian bonds during the Argentine crisis", Preprint, Instituto Argentino de Matemática, Buenos Aires

[4] Cané de Estrada, M.; Cortina, E.; Ferro Fontán, C; Di Fiori, J. (2005) "Pricing of defaultable bonds with log-normal spread: development of the model and an application to Argentinean and Brazilian bonds during the Argentine crisis", Review of Derivatives Research 8(1): 40-60.

[5] Hui, C.H.; Lo, C.F. (2002) "Valuation Model of Defaultable Bond Values in Emerging Markets", Asia-Pacific Financial Markets 9(1): 45-60.

[6] Lo, C.F.; Hui, C.H. (2000) "Valuation of defaultable bonds using signaling process An extension", Preprint, Chinese University of Hong Kong, Hong Kong

[7] Sukhomlin, N. (2006) "Conservation law of strike price in the Black-Scholes model", Economía 17-18: 147-161, Universidad de los Andes, Venezuela.

[8] Sukhomlin, N.; Jacquinot, Ph. (2006) "Symmetries and conservation laws in the Black-Scholes model of financial markets", The XVth International Symposium on Mathematical Methods Applied to Sciences, 153-154. In: E. Piza \& J. Trejos (Eds.), The University of Costa Rica, Costa Rica.

[9] Sukhomlin, N.; Jacquinot, Ph. (2006) "Conservation laws and the Black-Scholes model", The International Conference on the Mathematics of Optimization and Decision Making, University of Antilles and Guyana, Point-à-Pitre, Guadeloupe, France.

[10] Sukhomlin, N.; Jacquinot, Ph. (2006) "Explicit formulation of the volatility in the Black-Scholes-Merton model: theory and application", Journal of Future Markets (in publication).

[11] Wilmott, P.; Howison, S.; Dewynne, J. (1995) The Mathematics of Financial Derivatives. Cambridge University press, Cambridge 